XIX.

\title{
A CASE OF PROBABLE INJURY TO THE JUGULAR BULB FOLLOWING MYRINGOTOMY IN AN INFANT TEN MONTHS OLD.*
}

\author{
By John Randolph Page, M. D.,
}

\section{NFW YoRK.}

This case was admitted to Dr. Duel's service at the Manhattan Eye, Ear and Throat Hospital last July, with a discharge from both ears of six weeks' duration, temperature $102.6^{\circ}$ and considerable digestive disturbance. The house surgeon, Dr. Gallivan, found both drum membranes bulging posteriorly, so he performed a double myringotomy, the baby being swathed and no anesthetic used. After the myringotomy on the left side a profuse hemorrhage occurred; so profuse that it was thought best to pack the external auditory canal to control it. After this the child bled from the nose for a few minutes. On removing the packing at the end of five or ten minutes no further hemorrhage occurred. The ears were irrigated every three hours and the child kept under observation. The temperature, after a slight rise, gradually fell to $100.4{ }^{\circ}$ but twenty-four hours later went to $105 .^{\circ}$ The attending physician, Dr. Sammis, reported some enteritis with the ear condition and ordered treatment accordingly, but the temperature continued to rise to $105^{\circ}$ every night, in spite of apparent improvement in the ears and in the intestinal condition.

Blood examination showed no malaria, 14,000 leucocytes, 74 per cent polynuclear neutrophiles, but sufficient quantity could not be obtained for culture. Possible infection of the left jugular bulb was thought of from the first, but the extreme youth and delicate state of the patient made us particularly loth to operate unnecessarily. On the third day after admission, however, as the temperature the night before had again

- Presented before the Section on Otology, New York Academy of Medicine, November 14, 1914. 
gone to 105 , $^{\circ}$ the child was sent to the operating room for the purpose of having her left jugular resected, although there was practically no discharge from either ear and the skin over the mastoids was thin and entirely free from edema. The intention was to open the mastoid merely to block off the sinus and prevent return thow of infection from the bulb, and then, as rapidly as possible, to ligate and resect the internal jugular. To our surprise, on incising the thin skin over the mastoid, we found the cortex of the bone darkened, and when this had been removed, the antrum and cellular structure over the knee of the sinus filled with creamy pus, cultivation from which showed streptococci. The extensive involvement wa: so unexpected that operation on the other mastoid was now considered rather than operation on the jugular, as the external signs in it were just as sufficient to warrant operation as they had been on the side just exposed. Accordingly, the right mastoid was opened and practically the same condition was revealed. There was no exposure of the sinus or dura. as sufficient cause for the septic temperature was thought to have been found. On the following morning the temperature reached $105.8^{\circ}$ and during the following night $105 .^{\circ}$ so the next day it was again decided to remove the left jugular. The sinus on this side was exposed and incised, but no attempt was made to demonstrate a flow of blood from the bulb. Because of the infant's tender age it was packed quickly after incision and obliterated without an appreciable loss of blood. The jugular was then excised, but not without delay and embarrassment. owing to the large number of lymphatic glands which are always present in the neck of a child of this age. although they were not very much enlarged nor apparent until after the incision was made. Nothing pathologic could be noticed macroscopically in the vein itself, and it appeared to fill readily. As the neck was naturally short, the whole exposure was hardly more than an inch. The vein was ligated and excised, filled with blood, but unfortunately was not properly preserved for examination. The temperature the following day did not go above $104,^{\circ}$ and after that it gradually subsided to normal, with an uninterrupted recovery of the patient.

Since Dr. McKernon in 1905 called attention to the occurrence of primary jugular bulb thrombosis in children with 
IN JURY TO JUGULAR BULB FOLLOWING MYRINGOTONY. 163

purulent otitis media, and the close relation anatomically of the bulb to the floor of the tympanum, a number of other men have reported similar cases. In a short search in literature I have found no case where injury to the bulb had been reported after myringotomy, although I know of an instance where injury to the bulb occurred during the radical operation. It seems singular on observing temporal bones from children of this age that such injuries are not more common after myringotomy, particularly as high bulbs with a little if any bony separation between them and the tympanum are not infrequently met with. There is, I think, reason to believe that there was injury to the bulb following the myringotomy in this case, and consequent infection of it, although there is of course no definite proof that this was the case. 